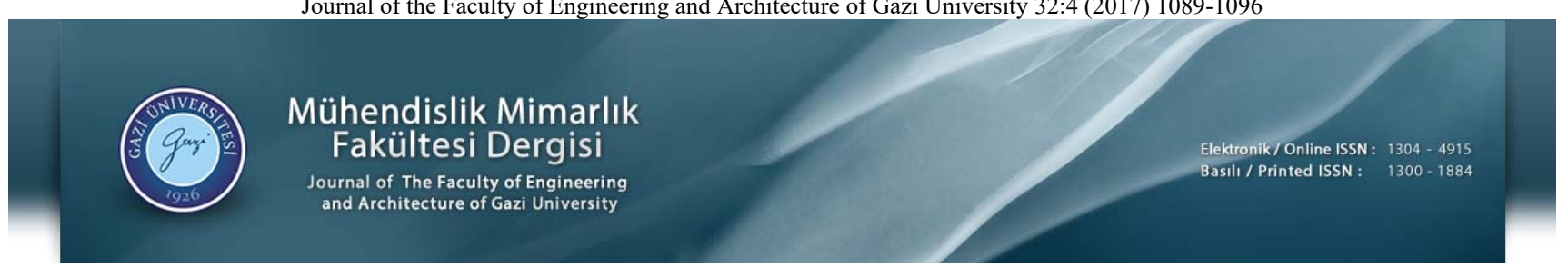

\title{
Tekil konumlardan geçen paralel robotlar için hareket planlamasında optimizasyon
}

Mustafa Özdemir *(1)

Marmara Üniversitesi, Mühendislik Fakültesi, Makine Mühendisliği Bölümü, Göztepe Yerleşkesi, 34722 Kadıköy, İstanbul, Türkiye

\section{Ö N E Ç I K A N L A R}

- Tekil konumlardan geçen paralel robotların hareket planlaması optimize edilmiştir

- Robotun ileri-geri hareketini önlemek için bir teorem geliştirilmiş ve ispatlanmıştır

- Geliştirilen yöntemin etkinliği sayısal bir örnek üzerinde gösterilmiştir

Makale Bilgileri

Geliş: 01.06.2016

Kabul: 30.07 .2017

DOI:

10.17341/gazimmfd.369403

Anahtar Kelimeler:

Paralel robot,

hareket planlamas1,

tekillik,

optimizasyon
ÖZET

Paralel robotlar seri robotlara kıyasla pek çok avantaja sahiptir. Bu nedenle, üretimden cerrahiye oldukça geniş bir alanda kullanılmaktadırlar. Ancak sahip oldukları Tip 2 tekillikler nedeniyle çalışma uzayları küçüktür. Bu probleme bir çözüm olarak literatürde tutarlı hareket planlaması önerilmiştir. Bu sayede bir paralel robot tekil konumlardan sorunsuzca geçebilmekte ve çalışma uzayının tamamını kullanabilmektedir. Ancak, bu yöntemin en büyük dezavantajı robotun uç noktasının yörüngesi üzerinde ileri-geri hareket etmesine yol açabilmesidir. Bu, verimlilik açısından kesinlikle istenmeyecek bir durumdur. Bu makalede söz konusu problemi önlemeye yönelik bir teorem geliştirilmiş ve ispatlanmıştır. Böylece tutarlı hareket planlaması yöntemi optimize edilerek paralel robotların daha verimli ve etkin bir şekilde kullanılmalarına olanak sağlanmıştır.

\section{Optimization in motion planning for parallel robots passing through singular positions}

\section{H I G H L I G H T S}

- Motion planning of parallel robots passing through singular positions is optimized

- A theorem is developed and proven for preventing back-and-forth motion of the robot

- The effectiveness of the developed method is illustrated on a numerical example

Article Info

Received: 01.06.2016

Accepted: 30.07 .2017

DOI:

10.17341/gazimmfd.369403

Keywords:

Parallel robot,

motion planning,

singularity,

optimization

\section{ABSTRACT}

Compared to serial robots, parallel robots have many advantages. For this reason, they are used in a wide area, from manufacturing to surgery. However, due to their Type 2 singularities, their workspace is small. As a solution to this problem, consistent motion planning has been proposed in the literature. In this manner a parallel robot can smoothly pass through singular positions, and the whole workspace can be utilized. But the biggest disadvantage of this method is that it can result in back-and-forth motion of the robot end-point along its trajectory. This is a totally undesirable situation from an efficiency perspective. In this article, a theorem is developed and proven to avoid the said problem. Thus, more efficient and effective use of parallel robots is enabled by optimizing the consistent motion planning method. 


\section{GİRIŞ (INTRODUCTION)}

Paralel robotlar, hassas konumlandırma kabiliyetleri [1, 2], yüksek hız [3] ve yüksek yük taşıma kapasiteleri [1, 4] gibi çeşitli avantajları sayesinde başta uçuş $[1]$ ve sürüş $[5,6]$ simülatörleri, yüksek hassasiyetli işleme tezgahları [7], tıbbi robotik $[8,9]$ ve robotik cerrahi $[10,11]$ olmak üzere geniş bir kullanım alanına sahiptirler. Ancak bu robotların en büyük dezavantajı çalışma uzaylarının içerisinde yer alan Tip 2 tekilliklerdir [12]. Bu tekillikler robotun bir ya da birden fazla serbestlik derecesi kazanmas1 [12], gerekli kuvvetlerin sonsuza raksaması ile birlikte eyleyicilerinin doyması ve sonuç olarak hareket kontrolünü kaybetmesi ile karakterize edilirler [13, 14].

Paralel robotların Tip 2 tekil konumlardan kontrollü bir şekilde geçerek çalışma uzaylarının tamamını kullanabilmelerini sağlamak için, hareketli platformun hız ve ivmesi, hareket denklemleri geçilmek istenen tekil konumda tutarlı olacak şekilde planlanmalıdır [15, 16]. Jui ve Sun [17] bu prensibe dayanan ve eyleyici limitlerini de göz önüne alan bir algoritma üzerinde çalışmışlardır. Söz konusu prensip literatürde ayrıca fiziksel anlamı bakımdan irdelenmiş [18, 19], ve esnek eklemli [20] ve esnek uzuvlu [21] paralel robotlara da uyarlanmıştır. Tutarlı hareket planlamasına alternatif olarak geliştirilmiş tekilliklere karşı gürbüz dengeleme [22] ve tekilliklerde tutarlılığa sahip yükleme $[23,24]$ yöntemleri de mevcuttur. Paralel robotların tekil konumlardan geçebilmesi için tutarlılık koşuluna ek olarak sağlanması gereken diğer koşullar Özdemir [25] tarafindan sunulmuştur. $\mathrm{Bu}$ çalışmaya da konu olan tutarlı hareket planlaması yöntemi, sözü edilen problemin çözümüne dair önemli bir adım olmakla beraber bir dezavantaja da sahiptir. Hareketli platform için tekil konumda robotun dinamik denklemlerinin tutarlı olmasını sağlayacak şekilde tayin edilen hız ve ivme, robotun uç noktasının ileri-geri hareket etmesine ve de enerji verimsizliğine yol açabilir. Verimsiz enerji tüketimi robotlarda önemli bir sorun teşkil etmektedir [26]. Her ne kadar böyle bir hareket ihtimaline daha önce Ider [15] tarafından dikkat çekilmiş olsa da bunu önleyici herhangi bir yöntem bulunmamaktadır. Literatürdeki bu eksikliği giderebilmek amacıyla, bu makalede Tip 2 tekil konumlardan geçen bir paralel robotun tutarlı hareket planlamasında robotun uç noktasının ileri-geri hareketinin önlenmesine dair bir teorem tasarlanmış ve ispatlanmıştır.

\section{PROBLEMIN TANIMI (DEFINITION OF THE PROBLEM)}

Robotun uç noktası $P$ olsun. Zaman $t$ ile gösterilirse, verilen bir $A x y z$ sabit koordinat sisteminde $P$ noktasının konumu $\mathbf{r}(t)=\left[\begin{array}{lll}x(t) & y(t) & z(t)\end{array}\right]^{\mathrm{T}}$ vektörü ile tanımlanır. Bir değişkenin, hareketin başlangıç ve bitiş noktalarındaki değerlerini sırasıyla 0 ve $f$ alt simgeleri ile gösterelim. Herhangi iki nokta arasındaki en kısa yol, bu noktaları birbirine bağlayan doğru parçası olduğundan robotun uç noktasının istenen herhangi iki $P_{0}$ ve $P_{f}$ noktası arasındaki doğru parçası boyunca hareket edeceği varsayılabilir. $\mathrm{Bu}$ durumda $P$ noktasının yörüngesi parametrik formda Eş. 1-3'te olduğu gibi ifade edilebilir.

$$
\begin{aligned}
& x(t)=x_{0}+f(t)\left(x_{f}-x_{0}\right) \\
& y(t)=y_{0}+f(t)\left(y_{f}-y_{0}\right) \\
& z(t)=z_{0}+f(t)\left(z_{f}-z_{0}\right)
\end{aligned}
$$

Bu denklemlerde $f$ zamana bağlı bir fonksiyon olup hareket planlaması sırasında belirlenecektir. $\mathrm{Bu}$ amaçla $f$ fonksiyonunun hareketin başlangıç ve bitiş anlarındaki değerleri sırasıyla Eş. 4 ve Eş. 5 'teki gibi yazılır.

$$
\begin{aligned}
& f(0)=0 \\
& f\left(t_{f}\right)=1
\end{aligned}
$$

Hareketin başlangıç ve bitişinde hız ve ivmenin sıfır olduğu kabul edilirse Eş. 6-9 elde edilir.

$$
\begin{aligned}
& \dot{f}(0)=0 \\
& \ddot{f}(0)=0 \\
& \dot{f}\left(t_{f}\right)=0 \\
& \ddot{f}\left(t_{f}\right)=0
\end{aligned}
$$

Yörünge üzerinde $f=f_{s}$ olduğunda robot Tip 2 bir tekil konuma gelmiş olsun. Robotun bu konuma geldiği zaman $t_{s}$ ile gösterilirse, $f$ fonksiyonunun bu andaki değeri Eş. 10 ile ifade edilir.

$$
f\left(t_{s}\right)=f_{s}
$$

Burada olduğu gibi makalenin geri kalan kısımlarında da $s$ alt simgesi bir değişkenin, robot tekil konumda iken alacağ 1 değeri göstermek için kullanılmıştır. Robot tekil konumdayken uç noktasının hız ve ivmesi ile ilgili olarak sırasıyla Eş. 11 ve Eş. 12 yazılır.

$$
\begin{gathered}
\dot{f}\left(t_{s}\right)=\dot{f}_{s} \\
\ddot{f}\left(t_{s}\right)=\ddot{f_{s}}
\end{gathered}
$$

Verilen $t_{f}, t_{s}, f_{s}$ ve seçilen $\dot{f}_{s}$ ve $\ddot{f}_{s}$ değerleri için Eş. 4-12'yi sağlayacak sürekli bir $f$ fonksiyonu sekizinci dereceden bir polinomla Eş. 13'te olduğu gibi ifade edilebilir. 


$$
f(t)=\sum_{n=0}^{8} a_{n} t^{n}
$$

İlgili polinom katsayıları Eş. 14-20’de verilmiştir.

$$
\begin{aligned}
& a_{0}=a_{1}=a_{2}=0 \\
& a_{3}=\frac{\left(t_{f} / t_{s}\right)^{3}}{2\left(t_{f}-t_{s}\right)^{5}} \times \\
& {\left[4\left(14 t_{s}^{2}-16 t_{f} t_{s}+5 t_{f}^{2}\right) f_{s}-\right.} \\
& 2\left(7 t_{s}^{2}-11 t_{f} t_{s}+4 t_{f}^{2}\right) t_{s} \dot{f}_{s}+\left(t_{f}-t_{s}\right)^{2} t_{s}^{2} \ddot{f}_{s}- \\
& \left.4\left(5 t_{s}^{2}-16 t_{f} t_{s}+14 t_{f}^{2}\right)\left(t_{s} / t_{f}\right)^{6}\right] \\
& a_{4}=\frac{t_{f}{ }^{2}}{2 t_{s}{ }^{4}\left(t_{f}-t_{s}\right)^{5}} \times \\
& {\left[-6\left(28 t_{s}^{3}-16 t_{f} t_{s}^{2}-7 t_{f}^{2} t_{s}+5 t_{f}^{3}\right) f_{s}+\right.} \\
& 2\left(21 t_{s}^{3}-20 t_{f} t_{s}^{2}-8 t_{f}^{2} t_{s}+7 t_{f}^{3}\right) t_{s} \dot{f}_{s}- \\
& \left(3 t_{s}^{3}-4 t_{f} t_{s}^{2}-t_{f}^{2} t_{s}+2 t_{f}^{3}\right) t_{s}^{2} \ddot{f}_{s}+ \\
& \left.6\left(5 t_{s}^{3}-7 t_{f} t_{s}^{2}-16 t_{f}^{2} t_{s}+28 t_{f}^{3}\right)\left(t_{s} / t_{f}\right)^{6}\right] \\
& a_{5}=\frac{t_{f}}{2 t_{s}^{5}\left(t_{f}-t_{s}\right)^{5}} \times \\
& {\left[12\left(14 t_{s}^{4}+8 t_{f} t_{s}^{3}-17 t_{f}{ }^{2} t_{s}^{2}+4 t_{f}{ }^{3} t_{s}+t_{f}{ }^{4}\right) f_{s}-\right.} \\
& 6\left(7 t_{s}^{4}+2 t_{f} t_{s}^{3}-14 t_{f}{ }^{2} t_{s}^{2}+4 t_{f}{ }^{3} t_{s}+t_{f}{ }^{4}\right) t_{s} \dot{f}_{s}+ \\
& \left(3 t_{s}^{4}-8 t_{f}^{2} t_{s}^{2}+4 t_{f}^{3} t_{s}+t_{f}^{4}\right) t_{s}^{2} \ddot{f}_{s}- \\
& \left.12\left(t_{s}^{4}+4 t_{f} t_{s}^{3}-17 t_{f}{ }^{2} t_{s}^{2}+8 t_{f}{ }^{3} t_{s}+14 t_{f}^{4}\right)\left(t_{s} / t_{f}\right)^{6}\right]
\end{aligned}
$$$$
a_{6}=\frac{1}{2 t_{s}^{5}\left(t_{f}-t_{s}\right)^{5}} \times
$$$$
\left[-4\left(14 t_{s}^{4}+56 t_{f} t_{s}^{3}-40 t_{f}^{2} t_{s}^{2}-9 t_{f}^{3} t_{s}+9 t_{f}^{4}\right) f_{s}+\right.
$$$$
2\left(7 t_{s}^{4}+28 t_{f} t_{s}^{3}-38 t_{f}{ }^{2} t_{s}^{2}-6 t_{f}^{3} t_{s}+9 t_{f}^{4}\right) t_{s} \dot{f}_{s}-
$$$$
\left(t_{s}^{4}+4 t_{f} t_{s}^{3}-8 t_{f}^{2} t_{s}^{2}+3 t_{f}^{4}\right) t_{s}^{2} \ddot{f}_{s}+
$$$$
\left.4\left(9 t_{s}^{4}-9 t_{f} t_{s}^{3}-40 t_{f}{ }^{2} t_{s}^{2}+56 t_{f}{ }^{3} t_{s}+14 t_{f}^{4}\right)\left(t_{s} / t_{f}\right)^{5}\right]
$$$$
a_{7}=\frac{1}{2 t_{s}^{5}\left(t_{f}-t_{s}\right)^{5}} \times
$$$$
\left[12\left(8 t_{s}^{3}+2 t_{f} t_{s}^{2}-8 t_{f}^{2} t_{s}+3 t_{f}^{3}\right) f_{s}-\right.
$$$$
2\left(13 t_{s}^{3}-2 t_{f} t_{s}^{2}-20 t_{f}^{2} t_{s}+9 t_{f}^{3}\right) t_{s} \dot{f}_{s}+
$$$$
\left(2 t_{s}^{3}-t_{f} t_{s}^{2}-4 t_{f}^{2} t_{s}+3 t_{f}^{3}\right) t_{s}^{2} \ddot{f}_{s}-
$$$$
\left.12\left(3 t_{s}^{3}-8 t_{f} t_{s}^{2}+2 t_{f}^{2} t_{s}+8 t_{f}^{3}\right)\left(t_{s} / t_{f}\right)^{5}\right]
$$

$$
\begin{aligned}
a_{8} & =\frac{1}{2 t_{s}^{5}\left(t_{f}-t_{s}\right)^{5}} \times \\
& {\left[-6\left(7 t_{s}^{2}-7 t_{f} t_{s}+2 t_{f}^{2}\right) f_{s}+\right.} \\
& 6\left(2 t_{s}^{2}-3 t_{f} t_{s}+t_{f}^{2}\right) t_{s} \dot{f}_{s}-\left(t_{f}-t_{s}\right)^{2} t_{s}^{2} \ddot{f}_{s}+ \\
& \left.6\left(2 t_{s}^{2}-7 t_{f} t_{s}+7 t_{f}^{2}\right)\left(t_{s} / t_{f}\right)^{5}\right]
\end{aligned}
$$

$$
\begin{aligned}
& h_{2}=\frac{1}{2 t_{s}^{5}\left(t_{f}-t_{s}\right)^{5}} \times \\
& {\left[12\left(5 t_{f}^{3}-42 t_{f} t_{s}^{2}+56 t_{s}^{3}\right) f_{s}-\right.} \\
& 2\left(15 t_{f}^{3}+4 t_{f}^{2} t_{s}-110 t_{f} t_{s}^{2}+91 t_{s}^{3}\right) t_{s} \dot{f}_{s}+ \\
& \left(5 t_{f}^{3}+4 t_{f}{ }^{2} t_{s}-23 t_{f} t_{s}^{2}+14 t_{s}{ }^{3}\right) t_{s}{ }^{2} \ddot{f}_{s}- \\
& \left.12 t_{s}\left(70 t_{f}^{2}-72 t_{f} t_{s}+21 t_{s}^{2}\right)\left(t_{s} / t_{f}\right)^{5}\right] \\
& h_{3}=\frac{4}{t_{s}^{5}\left(t_{f}-t_{s}\right)^{5}} \times \\
& {\left[-6\left(2 t_{f}^{2}-7 t_{f} t_{s}+7 t_{s}^{2}\right) f_{s}+\right.} \\
& 6\left(t_{f}{ }^{2}-3 t_{f} t_{s}+2 t_{s}^{2}\right) t_{s} \dot{f}_{s}-\left(t_{f}-t_{s}\right)^{2} t_{s}^{2} \ddot{f}_{s}+ \\
& \left.6\left(7 t_{f}^{2}-7 t_{f} t_{s}+2 t_{s}^{2}\right)\left(t_{s} / t_{f}\right)^{5}\right] \\
& h_{0}=\frac{3 t_{f}}{2 t_{s}^{3}\left(t_{f}-t_{s}\right)^{5}} \times \\
& {\left[4\left(5 t_{f}^{2}-16 t_{f} t_{s}+14 t_{s}^{2}\right) f_{s}-\right.} \\
& 2\left(4 t_{f}{ }^{2}-11 t_{f} t_{s}+7 t_{s}{ }^{2}\right) t_{s} \dot{f}_{s}+\left(t_{f}-t_{s}\right)^{2} t_{s}{ }^{2} \ddot{f}_{s}- \\
& h_{1}=\frac{1}{t_{s}^{4}\left(t_{f}-t_{s}\right)^{5}} \times \\
& {\left[-12\left(5 t_{f}^{3}-12 t_{f}^{2} t_{s}+14 t_{s}^{3}\right) f_{s}+\right.} \\
& \begin{array}{l}
14\left(2 t_{f}{ }^{3}-4 t_{f}{ }^{2} t_{s}-t_{f} t_{s}{ }^{2}+3 t_{s}{ }^{3}\right) t_{s} \ddot{f}_{s}- \\
\left(4 t_{f}{ }^{3}-5 t_{f}{ }^{2} t_{s}-2 t_{f} t_{s}{ }^{2}+3 t_{s}^{3}\right) t_{s}{ }^{2} \ddot{f}_{s}+
\end{array} \\
& \left.12 t_{s}\left(28 t_{f}^{2}-30 t_{f} t_{s}+9 t_{s}^{2}\right)\left(t_{s} / t_{f}\right)^{5}\right] \\
& \begin{array}{l}
\dot{f}_{s}+ \\
\left.t_{s}^{5}\right] \\
t_{s}^{2} \ddot{f}+
\end{array}
\end{aligned}
$$

Tip 2 bir tekil konumdan geçen bir yörüngenin robot tarafından izlenebilmesi için $\dot{f}_{s}$ ve $\ddot{f}_{s}$ hareket parametrelerinin (uç noktasınin yörünge bölümde bu problemi önlemeye yönelik bir teorem

\section{PROBLEMIN ÇÖZÜMÜ}

Bu bölümde, Tip 2 bir tekil konumdan geçecek bir paralel 位 noktasının koşul aşağıdaki teorem ile verilmiștir. Teorem. Bölüm 2'de $f$ de ğereri verilmis ve $\dot{f}$ v $\dddot{f}_{s}$ değerleri robotun dinamik denklemleri geçilmek istenen Tip 2 tekil konumda tutarlı olacak şekilde seçilmiş olsun. Bu değerler kullanılarak aşağıda tanımlanan dört katsayı hesaplansın (Eş. 21-24). 
Eğer aşağıda verilen dört bilinmeyenli dört denklemden oluşan denklem sisteminin en az bir reel çözümü varsa Eş. 13-20 ile verilen $f$ fonksiyonu $\left(0, t_{f}\right)$ aralığında azalmayan bir fonksiyondur (Eş. 25-28).

$$
\begin{aligned}
& x_{1}^{2} t_{f}=h_{0} \\
& x_{2}^{2}-x_{1}^{2}+2 x_{1} x_{3} t_{f}=h_{1} \\
& 2 x_{2} x_{4}-2 x_{1} x_{3}+x_{3}^{2} t_{f}=h_{2} \\
& x_{4}{ }^{2}-x_{3}{ }^{2}=h_{3}
\end{aligned}
$$

İspat: Lagrange'ın ortalama değer teoreminin bir sonucu olarak [27], eğer $f$ fonksiyonunun türevi $\left(0, t_{f}\right)$ aralığında hiçbir zaman negatif bir değer almıyorsa $f$ bu aralıkta azalmayan bir fonksiyondur. $\mathrm{Bu}$ koşulu daha detaylı ifade edebilmek için $g=\dot{f}(t)$ fonksiyonu Eş. 29'daki gibi yazılabilir.

$$
g(t)=3 a_{3} t^{2}+4 a_{4} t^{3}+5 a_{5} t^{4}+6 a_{6} t^{5}+7 a_{7} t^{6}+8 a_{8} t^{7}
$$

Eş. 6-9 göstermektedir ki $t=0$ ve $t=t_{f}$ kökleri $g$ fonksiyonunun çift katlı kökleridir. $\mathrm{Bu}$ tespitle birlikte Eş. 29 yeniden düzenlenerek Eş. 30 elde edilebilir.

$$
g(t)=t^{2}\left(t-t_{f}\right)^{2} h(t)
$$

Yukarıdaki eşitlikte görülen $h$ fonksiyonu, teoremde tanımlı $h_{0}, h_{1}, h_{2}$ ve $h_{3}$ katsayıları kullanılarak Eş. 31 'deki gibi ifade edilebilir.

$$
h(t)=h_{0}+h_{1} t+h_{2} t^{2}+h_{3} t^{3}
$$

Eş. 30'da yer alan $t^{2}$ ve $\left(t-t_{f}\right)^{2}$ çarpanlarının hiçbir zaman negatif bir değer alamayacağını göz önüne alarak, $h$ fonksiyonunun $\left[0, t_{f}\right]$ aralığında negatif herhangi bir değer almaması durumunda $\dot{f}$ fonksiyonun da aynı aralıkta hiçbir zaman negatif olmayacağı sonucuna varılır.

Brickman ve Steinberg [28] tarafindan verilen Teorem 3'e göre, eğer $h$ fonksiyonu $\left[0, t_{f}\right]$ aralı̆̆ında hiçbir zaman negatif bir değer almıyorsa Eş. 32'yi sağlayacak şekilde $p(t)$ ve $q(t)$ reel polinomları bulunabilir.

$h(t)=[p(t)]^{2} t+[q(t)]^{2}\left(t_{f}-t\right)$

$h$ fonksiyonu üçüncü dereceden bir polinom fonksiyonu olduğu için $p$ ve $q$ birinci dereceden birer polinomdur. Buna göre, $p$ ve $q$ polinomları sırasıyla Eş. 33 ve Eş. 34'de olduğu gibi yazılabilir.

$$
\begin{aligned}
& p(t)=p_{0}+p_{1} t \\
& q(t)=q_{0}+q_{1} t
\end{aligned}
$$

Eş. 31, Eş. 33 ve Eş. 34, Eş. 32'de yerlerine konulduğunda, Eş. 35 elde edilebilir.

$$
\begin{aligned}
& h_{0}+h_{1} t+h_{2} t^{2}+h_{3} t^{3}=q_{0}^{2} t_{f}+\left(p_{0}^{2}-q_{0}^{2}+2 q_{0} q_{1} t_{f}\right) t+ \\
& \left(2 p_{0} p_{1}-2 q_{0} q_{1}+q_{1}^{2} t_{f}\right) t^{2}+\left(p_{1}^{2}-q_{1}^{2}\right) t^{3}
\end{aligned}
$$

Yukarıdaki denklemin her iki tarafında yer alan aynı dereceli terimlerin katsayıları birbirine eşitlenerek, Eş. 36-39 yazılabilir.

$$
\begin{aligned}
& q_{0}^{2} t_{f}=h_{0} \\
& p_{0}^{2}-q_{0}^{2}+2 q_{0} q_{1} t_{f}=h_{1} \\
& 2 p_{0} p_{1}-2 q_{0} q_{1}+q_{1}^{2} t_{f}=h_{2}
\end{aligned}
$$

$p_{1}^{2}-q_{1}^{2}=h_{3}$

Eğer Eş. 36-39'dan oluşan denklem sisteminin en az bir reel çözümü varsa $h$ ve dolayısıyla $\dot{f}$ fonksiyonları $\left[0, t_{f}\right]$ aralığında asla negatif olmaz. Böylece, $f$ fonksiyonunun $\left(0, t_{f}\right)$ aralığında azalmayan bir fonksiyon olduğu garantilenir ve Eş. 1-3'e göre robotun uç noktasının ileri-geri hareketi önlenir.

\section{SAYISAL ÖRNEK (NUMERICAL EXAMPLE)}

$\mathrm{Bu}$ bölümde, bir önceki bölümde geliştirilen teoremin uygulaması iki serbestlik dereceli bir 2-RRR düzlemsel paralel robot üzerinde açıklanacaktır. Söz konusu robot Şekil 1 'de gösterilmiştir.

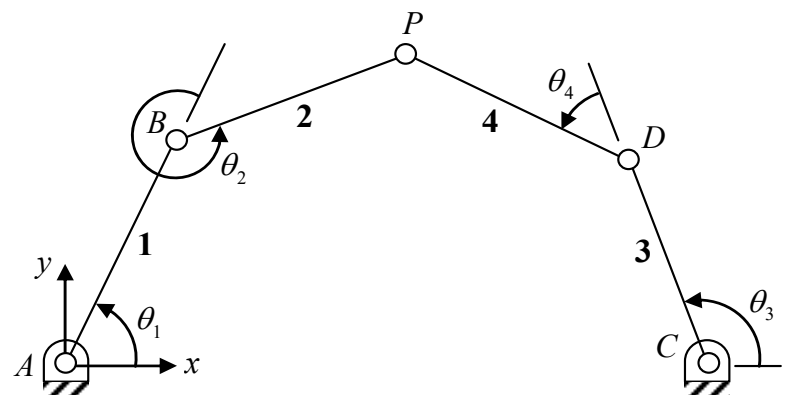

Şekil 1. İki serbestlik dereceli 2-RRR düzlemsel paralel robot (Two-degree-of-freedom 2-RRR planar parallel robot)

Robotun yatay düzlemde çalıştığı ve hareketli uzuvlarının $L_{k}$ uzunluğunda ve $m_{k}$ kütleli $(k=1,2,3,4)$ ince, düzgün ve türdeş çubuklar olduğu varsayılmıştır. $A$ ve $C$ eklemleri sirasıyla $T_{1}$ ve $T_{2}$ motor torkları ile hareket ettirilmektedir. Robot parametrelerinin sayısal değerleri şöyledir: $L_{0}=|A C|=0,9377 \mathrm{~m}, L_{1}=0,6 \mathrm{~m}, L_{2}=L_{4}=0,2 \mathrm{~m}, L_{3}=0,5 \mathrm{~m}$, $m_{1}=1,2 \mathrm{~kg}, m_{2}=m_{4}=0,4 \mathrm{~kg}$ ve $m_{3}=1 \mathrm{~kg}$. Robotun dinamik denklemleri Eş. 40’taki gibidir. 


$$
\begin{aligned}
& {\left[\begin{array}{llll}
1 & 0 & H_{13} & H_{14} \\
0 & 0 & H_{23} & H_{24} \\
0 & 1 & H_{33} & H_{34} \\
0 & 0 & H_{43} & H_{44}
\end{array}\right]\left[\begin{array}{c}
T_{1} \\
T_{2} \\
\lambda_{1} \\
\lambda_{2}
\end{array}\right]=} \\
& {\left[\begin{array}{cccc}
M_{11} & M_{12} & 0 & 0 \\
M_{21} & M_{22} & 0 & 0 \\
0 & 0 & M_{33} & M_{34} \\
0 & 0 & M_{43} & M_{44}
\end{array}\right]\left[\begin{array}{l}
\ddot{\theta}_{1} \\
\ddot{\theta}_{2} \\
\ddot{\theta}_{3} \\
\ddot{\theta}_{4}
\end{array}\right]+\left[\begin{array}{c}
N_{1} \\
N_{2} \\
N_{3} \\
N_{4}
\end{array}\right]}
\end{aligned}
$$

Burada $\lambda_{1}$ ve $\lambda_{2}$ Lagrange çarpanları olup denklem sisteminde yer alan matris ve vektör elemanları Eş. 41-60’ta verilmiştir.

$$
\begin{aligned}
& H_{13}=-L_{1} \sin \theta_{1}-L_{2} \sin \left(\theta_{1}+\theta_{2}\right) \\
& H_{14}=L_{1} \cos \theta_{1}+L_{2} \cos \left(\theta_{1}+\theta_{2}\right) \\
& H_{23}=-L_{2} \sin \left(\theta_{1}+\theta_{2}\right) \\
& H_{24}=L_{2} \cos \left(\theta_{1}+\theta_{2}\right) \\
& H_{33}=L_{3} \sin \theta_{3}+L_{4} \sin \left(\theta_{3}+\theta_{4}\right) \\
& H_{34}=-L_{3} \cos \theta_{3}-L_{4} \cos \left(\theta_{3}+\theta_{4}\right) \\
& H_{43}=L_{4} \sin \left(\theta_{3}+\theta_{4}\right) \\
& H_{44}=-L_{4} \cos \left(\theta_{3}+\theta_{4}\right) \\
& M_{11}=\frac{1}{3}\left(m_{1} L_{1}{ }^{2}+m_{2} L_{2}{ }^{2}\right)+m_{2} L_{1}\left(L_{1}+L_{2} \cos \theta_{2}\right) \\
& M_{12}=\frac{1}{6} m_{2} L_{2}\left(2 L_{2}+3 L_{1} \cos \theta_{2}\right) \\
& M_{21}=\frac{1}{6} m_{2} L_{2}\left(2 L_{2}+3 L_{1} \cos \theta_{2}\right) \\
& M_{22}=\frac{1}{3} m_{2} L_{2}^{2} \\
& M_{33}=\frac{1}{3}\left(m_{3} L_{3}{ }^{2}+m_{4} L_{4}{ }^{2}\right)+m_{4} L_{3}\left(L_{3}+L_{4} \cos \theta_{4}\right) \\
& M_{34}=\frac{1}{6} m_{4} L_{4}\left(2 L_{4}+3 L_{3} \cos \theta_{4}\right) \\
& M_{43}=\frac{1}{6} m_{4} L_{4}\left(2 L_{4}+3 L_{3} \cos \theta_{4}\right)
\end{aligned}
$$

$$
\begin{aligned}
& M_{44}=\frac{1}{3} m_{4} L_{4}^{2} \\
& N_{1}=-\frac{1}{2} m_{2} L_{1} L_{2}\left(2 \dot{\theta}_{1}+\dot{\theta}_{2}\right) \dot{\theta}_{2} \sin \theta_{2} \\
& N_{2}=\frac{1}{2} m_{2} L_{1} L_{2} \dot{\theta}_{1}^{2} \sin \theta_{2} \\
& N_{3}=-\frac{1}{2} m_{4} L_{3} L_{4}\left(2 \dot{\theta}_{3}+\dot{\theta}_{4}\right) \dot{\theta}_{4} \sin \theta_{4} \\
& N_{4}=\frac{1}{2} m_{4} L_{3} L_{4} \dot{\theta}_{3}^{2} \sin \theta_{4}
\end{aligned}
$$

Mekanizmanın Tip 2 tekil konfigürasyonları Eş. 61 ile ifade edilebilir [16].

$$
\begin{aligned}
& \operatorname{det} \mathrm{H}=\left|\begin{array}{llll}
1 & 0 & H_{13} & H_{14} \\
0 & 0 & H_{23} & H_{24} \\
0 & 1 & H_{33} & H_{34} \\
0 & 0 & H_{43} & H_{44}
\end{array}\right| \\
& =-L_{2} L_{4} \sin \left(\theta_{1}+\theta_{2}-\theta_{3}-\theta_{4}\right)=0
\end{aligned}
$$

Eş. 61'in çözümü Eş. 62'deki gibidir.

$\theta_{1}+\theta_{2}-\theta_{3}-\theta_{4}= \pm n \pi, \quad n=0,1,2, \cdots$

Robotun uç noktası olan $P^{\prime} \operatorname{nin}\left(x_{0}, y_{0}\right)=(0,45,0,45) \mathrm{m}$ noktasından $\left(x_{f}, y_{f}\right)=(0,5,0,5) \mathrm{m}$ noktasına $t_{f}=1 \mathrm{~s}$ 'de ulaşmak üzere doğrusal bir yörünge izleyeceği varsayılmıştır. Görev, sıfır hız ve ivmeyle başlayacak ve bitecektir. Bu görev tanımına uygun bir yörünge fonksiyonu Eş. 63'te verilmiştir.

$$
f(t)=10 t^{3}-15 t^{4}+6 t^{5}
$$

$\mathrm{Bu}$ görevin yerine getirilmesi için $t_{s}=0,5 \mathrm{~s}$ 'de $\left(x_{s}, y_{s}\right)=(0,475$, $0,475) \mathrm{m}$ tekillik noktasından $\left(f_{s}=0,5\right)$ geçilmesi gerekmektedir. Eş. 63 ile tanımlanan yörünge fonksiyonunu gerçekleştirmek için gerekli olan motor torkları Şekil 2'de görülmektedir. Dinamik model tekil konumda tutarsız olduğu için bu konum etrafinda gerekli torkların büyüklükleri sonsuza 1raksamaktadır. $\mathrm{Bu}$ 1raksama literatürde verilen sonuçlardan beklendiği gibidir [16, 18]. Geçilmek istenen tekil konumda robotun eklem açılarının alacağ1 değerler şöyledir: $\theta_{1}=61,9^{\circ}, \theta_{2}=282,4^{\circ}, \theta_{3}=122,7^{\circ}$ ve $\theta_{4}=41,6^{\circ}$.

Tekil konumda $\mathrm{H}$ matrisinin ikinci ve dördüncü satırları arasındaki ilişki Eş. 64 ile gösterilebilir.

$H_{2 j}-\frac{L_{2}}{L_{4}} H_{4 j}=0, \quad j=3,4$ 


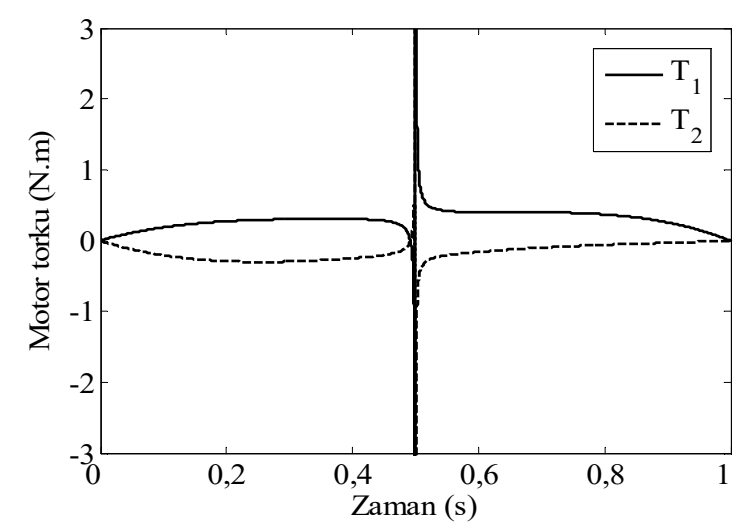

Şekil 2. Tutarsız bir yörünge için gerekli motor torkları (Required motor torques for an inconsistent trajectory)

Dinamik denklemlerin tutarlılı̆̆ için Eş. 40'ın sağ tarafında bulunan terimler arasında da tekil konumda aynı ilişki bulunmalıdır [16]. Yani Eş. 65 tekil konumda sağlanmalıdır.

$$
M_{21} \ddot{\theta}_{1}+M_{22} \ddot{\theta}_{2}+N_{2}-\frac{L_{2}}{L_{4}}\left(M_{43} \ddot{\theta}_{3}+M_{44} \ddot{\theta}_{4}+N_{4}\right)=0
$$

Rouché-Frobenius teoreminin [29] doğrudan bir sonucu olan bu tutarlılık koşulu gerek bu bölümde ele alınan mekanizma gerekse de genel bir paralel robot için literatürde detaylı olarak çalışılmıştır [16, 18]. Burada örnek olarak seçilen mekanizmanın tekilliklere karşı gürbüz dengelenmesi problemi ise Özdemir [30] tarafından çalışılmıştır. Kinematik ilişkiler ve yukarıdaki sayısal değerler kullanılarak Eş. 65 sadeleştirilebilir ve Eş. 66 elde edilebilir.

$$
\dot{f}_{s}^{2}-15,0513 \ddot{f_{s}}=0
$$

Eş. 66 ile verilen tutarlılık koşulunu sağlayan sonsuz sayıda $\left(\dot{f_{s}}, \ddot{f_{s}}\right)$ çözümü bulunmaktadır. Bunlardan bir tanesi olarak, $\dot{f}_{s}=7,5$ ve $\ddot{f}_{s}=3,7372$ seçilmiş olsun. Seçilen bu değerler için $f$ fonksiyonuna ait polinom katsayıları şöyledir: $a_{3}=-140,1022, \quad a_{4}=675,7155, \quad a_{5}=-1045,9, \quad a_{6}=512,5554$, $a_{7}=118,3645$ ve $a_{8}=-119,5911$. Paralel robotların tekil konumlardan geçebilmelerini mümkün kılmak için tutarlılık koşuluna ek olarak sağlanması gereken diğer koşullar Özdemir [25] tarafından verilmiştir. Bu örnek özelinde söz konusu ek koşul, tekillik anında $\mathrm{H}$ matrisinin determinantının zamana göre birinci türevinin sıfırdan farklı bir değere sahip olmasıdır. Yukarıda verilen tutarlı yörünge fonksiyonuyla bu ikinci koşul da sağlanmakta ve tekil konumdan geçilirken gerekli motor torkları Şekil 3'te görüldüğü gibi sonlu limitlere sahip olmaktadır. Ancak bu yörünge fonksiyonu tutarlı olmakla birlikte robot gitmesi gereken yönün tersi istikametinde hareketine başlamakta, bir süre bu şekilde ters yönde gittikten sonra olması gereken istikamete yönelmektedir. $\mathrm{Bu}$ istikamette ilerlerken belirlenen bitiş noktasının ötesine geçmekte ve bir süre sonra tekrar geriye doğru dönerek bu noktada durmaktadır. Bahsedilen problem Şekil 4'te görülebilir.

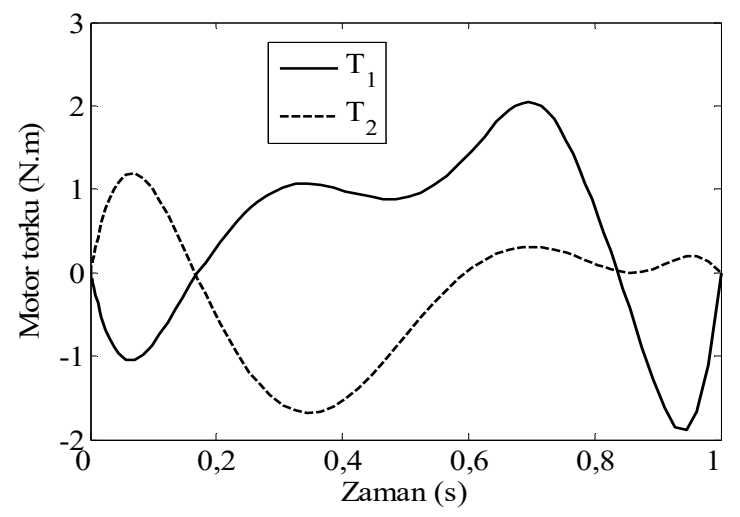

Şekil 3. $\dot{f_{s}}=7,5$ ve $\ddot{f_{s}}=3,7372$ olacak şekilde seçilen tutarlı yörünge için gerekli motor torkları (Required motor torques for the selected consistent trajectory such that $\dot{f}_{s}=7.5$ and $\ddot{f}_{s}=3.7372$ )

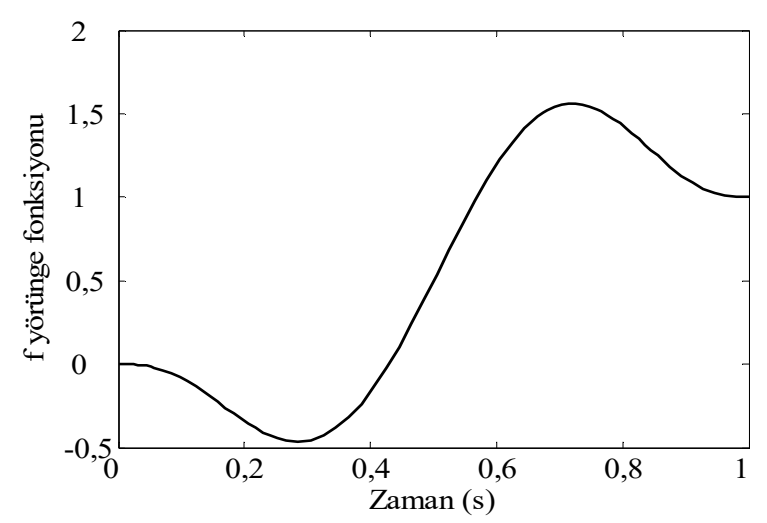

Şekil 4. $\dot{f}_{s}=7,5$ ve $\ddot{f_{s}}=3,7372$ olacak şekilde seçilen tutarlı yörünge fonksiyonunun grafiği

(Graph of the selected consistent trajectory function such that $\dot{f}_{s}=7.5$ and $\ddot{f}=3.7372$ )

Problemin çözümü için bu makalede geliştirilen teorem kullanılabilir. $\mathrm{Bu}$ teoremin uygulama adımları aynı örnek için aşağıda sunulmuştur.

Eş. 66 ile verilen tutarlılık koşulunu sağlayacak şekilde $\dot{f_{s}}=0,25$ ve $\ddot{f_{s}}=0,0042$ değerleri seçilmiş olsun.

Adım 1: Seçilen bu değerler için teoremde tanıml $h_{0}, h_{1}, h_{2}$ ve $h_{3}$ katsayıları şöyle hesaplanır: $h_{0}=186,0997$, $h_{1}=-728,7308, h_{2}=729,5945$ ve $h_{3}=-1,0630$.

Adım 2: Adım 1'de hesaplanan katsayılar kullanılarak, teoremde verilen denklem sistemi şöyle yazılır (Eş. 67-70):

$$
\begin{aligned}
& x_{1}^{2}=186,0997 \\
& x_{2}^{2}-x_{1}^{2}+2 x_{1} x_{3}=-728,7308 \\
& 2 x_{2} x_{4}-2 x_{1} x_{3}+x_{3}^{2}=729,5945 \\
& x_{4}{ }^{2}-x_{3}{ }^{2}=-1,0630
\end{aligned}
$$


Adım 3: Adım 2'de yazılan denklem sisteminin bir reel çözümü şöyle bulunabilir: $x_{1}=-13,6418, x_{2}=-9,6305$, $x_{3}=23,2878$ ve $x_{4}=23,265$. Böylece $\dot{f_{s}}=0,25$ ve $\ddot{f_{s}}=0,0042$ olacak şekilde seçilen yeni $f$ fonksiyonunun görev süresi boyunca azalmayan bir fonksiyon olduğu garantilenir.

Adım 4: Seçilen $\dot{f_{s}}=0,25$ ve $\ddot{f_{s}}=0,0042$ değerleri için Eş. $15-20$ kullanılarak yeni $f$ fonksiyonuna ait polinom katsay1ları şöyle hesaplanır: $a_{3}=62,0332, a_{4}=-275,2325, a_{5}=474,6312$, $a_{6}=-364,8305, a_{7}=104,5315$ ve $a_{8}=-0,1329$.

Bu yeni yörünge fonksiyonu ile hem Şekil 5'te görüldüğü üzere gerekli motor torklarının tekil konumdan geçerken sonlu limitlere sahip olması sağlanmakta hem de Şekil 6'dan anlaşılabileceği üzere robot herhangi bir ileri-geri hareketi yapmamaktadır (H matrisinin determinantının zamana göre birinci türevinin tekillik anında sıfırdan farklı bir değere sahip olması ek koşulu bu yeni yörünge fonksiyonu ile de sağlanmıştır).

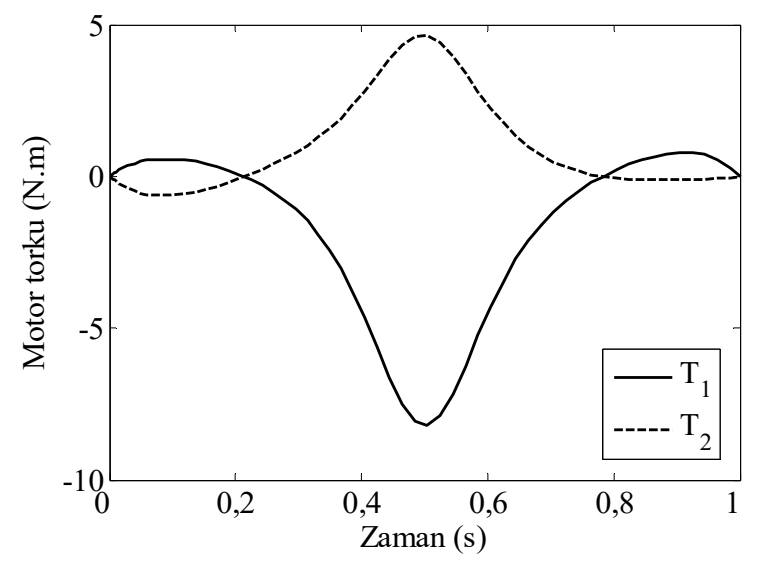

Şekil 5. $\dot{f_{s}}=0,25$ ve $\ddot{f_{s}}=0,0042$ olacak şekilde seçilen tutarl yörünge için gerekli motor torkları

(Required motor torques for the selected consistent trajectory such that $\dot{f}_{s}=0.25$ and $\ddot{f}_{s}=0.0042$ )

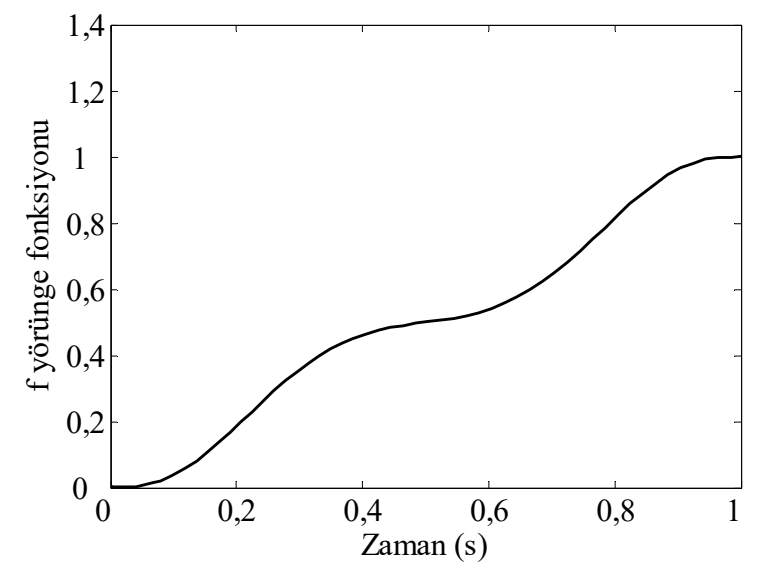

Şekil 6. $\dot{f_{s}}=0,25$ ve $\ddot{f_{s}}=0,0042$ olacak şekilde seçilen tutarlı yörünge fonksiyonunun grafiği (Graph of the selected consistent trajectory function such that $\dot{f}_{s}=0.25$ and $\left.\ddot{f_{s}}=0.0042\right)$

\section{SIMGELER (SYMBOLS)}

$\begin{array}{ll}\begin{array}{ll}\text { Axyz } \\ \text { det }\end{array} & \text { Sabit koordinat sistemi } \\ f & \text { Determinant } \\ P & \text { Yörünge fonksiyonu } \\ t & \text { Robotun uç noktası } \\ & \text { Zaman }\end{array}$

Alt Simgeler (Subscripts)

0 Bir değişkenin hareketin başlangıç noktasındaki değeri

$f \quad$ Bir değişkenin hareketin bitiş noktasındaki değeri

$s \quad$ Bir değişkenin robot tekil konumdayken aldığı değer

Üst Simgeler (Superscripts)

T Matrisin transpozesi

\section{SONUÇLAR (CONCLUSIONS)}

$\mathrm{Bu}$ makalede paralel robotların Tip 2 tekil konumlardan geçmesini sağlamak için literatürde kullanılan tutarlı hareket planlaması yönteminin optimizasyonu gerçekleştirilmiştir. $\mathrm{Bu}$ amaçla robotun uç noktasının izleyeceği yörüngenin doğrusal olduğu kabul edilerek bir teorem geliştirilmiş ve ispatlanmıştır. Geliştirilen teorem temel olarak robotun uç noktasının doğrusal yörüngesi üzerinde istenmeyecek şekilde ileri-geri hareket etmesini önlemeye yönelik bir koşul ortaya koymaktadır. $\mathrm{Bu}$ teoreme dayanan yeni yöntemin etkili bir şekilde uygulanabilirliğini göstermek için sayısal bir örnek de verilmiştir. $\mathrm{Bu}$ çalışmanın paralel robotların daha etkili ve verimli bir biçimde kullanılmasını sağlamak suretiyle literatüre önemli bir katkı sağlayacağına inanılmaktadır. Son olarak belirtilmelidir ki, model belirsizlikleri robotun planlanan hareketten sapmasina neden olur. Bunu önlemek için uygun bir kontrol sisteminin kullanılmas1 gerekmektedir [17, 31].

\section{KAYNAKLAR (REFERENCES)}

1. Dasgupta B., Mruthyunjaya T.S., The Stewart platform manipulator: a review, Mech. Mach. Theory, 35 (1), 1540, 2000.

2. Kılıçaslan S., Tracking control of elastic joint parallel robots via state-dependent Riccati equation, Turkish Journal of Electrical Engineering and Computer Sciences, 23 (2), 522-538, 2015.

3. Briot S., Bonev I.A., Are parallel robots more accurate than serial robots?, Transactions of the Canadian Society for Mechanical Engineering, 31 (4), 445-455, 2007.

4. Zhang D., Su X., Gao Z., Qian J., Design, analysis and fabrication of a novel three degrees of freedom parallel robotic manipulator with decoupled motions, Int. J. Mech. Mater. Des., 9 (3), 199-212, 2013.

5. Tseng H.-L., Fong I.-K., Implementation of a driving simulator based on a Stewart platform and computer 
graphics technologies, Asian J. Control, 2 (2), 88-100, 2000.

6. Zhang C., Zhang L., Kinematics analysis and workspace investigation of a novel 2-DOF parallel manipulator applied in vehicle driving simulator, Rob. Comput. Integr. Manuf., 29 (4), 113-120, 2013.

7. Zhang D., Gao Z., Su X., Li J., A comparison study of three degree-of-freedom parallel robotic machine tools with/without actuation redundancy, Int. J. Computer Integr. Manuf., 25 (3), 230-247, 2012.

8. Lessard S., Bigras P., Bonev I.A., A new medical parallel robot and its static balancing optimization, J. Med. Devices, 1 (4), 272-278, 2007.

9. Li Y., Xu Q., Design and development of a medical parallel robot for cardiopulmonary resuscitation, IEEE/ASME Trans. Mechatron., 12 (3), 265-273, 2007.

10. Nakano T., Sugita N., Ueta T., Tamaki Y., Mitsuishi M., A parallel robot to assist vitreoretinal surgery, International Journal of Computer Assisted Radiology and Surgery, 4 (6), 517-526, 2009.

11. Pile J., Simaan N., Modeling, design, and evaluation of a parallel robot for cochlear implant surgery, IEEE/ASME Trans. Mechatron., 19 (6), 1746-1755, 2014.

12. Gosselin C., Angeles J., Singularity analysis of closedloop kinematic chains, IEEE Transactions on Robotics and Automation, 6 (3), 281-290, 1990.

13. Choudhury P., Ghosal A., Singularity and controllability analysis of parallel manipulators and closed-loop mechanisms, Mech. Mach. Theory, 35 (10), 1455-1479, 2000.

14. Bandyopadhyay S., Ghosal A., Analysis of configuration space singularities of closed-loop mechanisms and parallel manipulators, Mech. Mach. Theory, 39 (5), 519-544, 2004.

15. Ider S.K., Singularity robust inverse dynamics of planar 2-RPR parallel manipulators, Proceedings of the Institution of Mechanical Engineers, Part C: Journal of Mechanical Engineering Science, 218 (7), 721-730, 2004.

16. Ider S.K., Inverse dynamics of parallel manipulators in the presence of drive singularities, Mech. Mach. Theory, 40 (1), 33-44, 2005.

17. Jui C.K.K., Sun Q., Path tracking of parallel manipulators in the presence of force singularity, J. Dyn. Syst. Meas. Contr., 127 (4), 550-563, 2005.
18. Briot S., Arakelian V., Optimal force generation in parallel manipulators for passing through the singular positions, Int. J. Rob. Res., 27 (8), 967-983, 2008.

19. Briot S., Pagis G., Bouton N., Martinet P., Degeneracy conditions of the dynamic model of parallel robots, Multibody Sys.Dyn., 37 (4), 371-412, 2016.

20. Briot S., Arakelian V., On the dynamic properties of rigid-link flexible-joint parallel manipulators in the presence of type 2 singularities, Journal of Mechanisms and Robotics, 2 (2), 021004, 2010.

21. Briot S., Arakelian V., On the dynamic properties of flexible parallel manipulators in the presence of type 2 singularities, Journal of Mechanisms and Robotics, 3 (3), 031009, 2011.

22. Özdemir M., Singularity robust balancing of parallel manipulators following inconsistent trajectories, Robotica, 34 (9), 2027-2038, 2016.

23. Özdemir M., Singularity-consistent payload locations for parallel manipulators, Mech. Mach. Theory, 97, 171$189,2016$.

24. Özdemir M., Dynamic analysis of planar parallel robots considering singularities and different payloads, Rob. Comput. Integr. Manuf., 46, 114-121, 2017.

25. Özdemir M., Removal of singularities in the inverse dynamics of parallel robots, Mech. Mach. Theory, 107, 71-86, 2017.

26. Kilıç A., Kapucu S., Design and construction of a modular reconfigurable robot module OMNIMO, Journal of the Faculty of Engineering and Architecture of Gazi University, 31 (3), 521-530, 2016.

27. Zorich V.A., Mathematical Analysis I, Translator: Cooke R., Springer, Germany, 2004.

28. Brickman L., Steinberg L., On nonnegative polynomials, The American Mathematical Monthly, 69 (3), 218-221, 1962.

29. López C.P., MATLAB Optimization Techniques, Apress, 2014.

30. Özdemir M., Spring balancing of a five-bar parallel manipulator in the presence of singularities, 2nd International Conference on Advances in Mechanical Engineering (ICAME2016), İstanbul-Türkiye, 354-357, 10-13 Mayis 2016.

31. Pagis G., Bouton N., Briot S., Martinet, P., Enlarging parallel robot workspace through Type-2 singularity crossing, Control Eng. Pract., 39, 1-11, 2015. 